\title{
Can Liposuction Technique Be Used as a Treatment Method in Cases of Mercury Injections?
}

\author{
S. S. Shirol ${ }^{1,2}$ (D) Prathamesh Chandrapattan ${ }^{3}$
}

Received: 15 March 2017 / Accepted: 5 May 2017 /Published online: 11 May 2017

(C) Association of Surgeons of India 2017

Sir,

We read the case report titled "Liposuction technique used as a treatment modality for suicide attempt by injection of mercury" published in your reputed journal [1] with lots of interest. We congratulate the authors Sai Suraj Kotera and his team for reporting an interesting and innovative idea of the application of liposuction in mercury poisoning. However, the whole report revolves around the clinical symptoms and the traditional way of management whereas little or no emphasis has been given to the exact technique of the application of liposuction.

Liposuction typically involves injection of a tumescent fluid, usually Ringer's lactate, and lignocaine with adrenaline with/without hyaluronidase followed by suction with various cannulae after waiting for about 20 $30 \mathrm{~min}$ [2]. Whether any fluid/s were infused for tumescence before application of liposuction, the contents of the fluids, the quantity of such fluid, the type and size of cannulae used, the pressure settings applied, details of the skin incision for cannulation, end point of stopping the suction, and anaesthesia details have not been described in the report.
If tumescent fluids were injected in this case, whether it could have caused systemic spread ofmercury and toxicity has not been discussed by the authors. If no such tumescent technique was used, it would just be suctioning of mercury and not liposuction technique and the title of the paper becomes inappropriate.

We would be delighted to hear more technical details from the authors.

\section{Compliance with Ethical Standards}

Conflict of Interest The authors declare that they have no conflict of interest.

\section{References}

1. Kotera SS, Shankar KM, Rajagopalan S (2016) Liposuction technique used as a treatment modality for suicide attempt by injection of mercury. Indian Journal of Surgery 78(5):411-413

2. Shirol SS (2016) Orange peel excision of gland: a novel surgical technique for treatment of gynecomastia. Ann Plast Surg 77(6): 615-619

\section{S. S. Shirol \\ ssshirol@yahoo.co.uk}

1 Department of Plastic Surgery, KIMS-Hubli, Hubli, India

2 Sampige Superspecialty Clinic, 1st floor, Divate Complex, Club Road, Hubli, Karnataka 580028, India

3 General Surgery, KIMS-Hubli, Hubli, India 